\title{
LA DIALÉCTICA EN LAS INVESTIGACIONES EDUCATIVAS
}

DIALECTICS IN EDUCATIONAL RESEARCHS

\author{
Volumen 11, Número 2
}

pp. 1-26

Este número se publicó el 30 de agosto de 2011

Emilio Alberto Ortiz Torres

La revista está indexada en los directorios:

LATINDEX, REDALYC, IRESIE, $\underline{\text { CLASE}}$ DIALNET, DOAJ, E-REVIST@S,

La revista está incluida en los sitios:

REDIE, RINACE, OEI, MAESTROTECA, PREAL, HUASCARAN, CLASCO 


\title{
LA DIALÉCTICA EN LAS INVESTIGACIONES EDUCATIVAS DIALECTICS IN EDUCATIONAL RESEARCHS
}

\section{Emilio Alberto Ortiz Torres ${ }^{1}$}

\begin{abstract}
Resumen: En las investigaciones educativas se refleja la dialéctica al manifestarse contradicciones en el objeto de estudio, que se presuponen y se niegan al unísono. Sin embargo, los principios y las categorías de la lógica dialéctica casi no son abordados, de manera explícita, en la formación de profesionales en investigación educativa, en los manuales elaborados, ni en la propia práctica inquisitoria. Por lo general, solo se resalta la importancia de las leyes y reglas de la lógica formal que considera a la contradicción como algo indeseable y que se debe eliminar en aras de conservar el rigor científico, cuando en realidad ambas no son excluyentes, por el contrario, su complementariedad deviene en una exigencia contemporánea para las investigaciones científicas. Por lo anterior, el objetivo de este ensayo es ofrecer varios argumentos y recomendaciones que contribuyen a la aplicación rigurosa, consecuente y sistemática de la lógica dialéctica en las investigaciones educativas. Además, puede servir de consulta para el diseño y el desarrollo de investigaciones en el campo educativo.
\end{abstract}

Palabras clave: LÓGICA DIALÉCTICA, CONTRADICCIÓN, INVESTIGACIÓN EDUCATIVA

Abstract: In educational research, dialectics is represented by contradictions in the object of study which are both assumed and negated. However, the principles and categories of dialectic logic are infrequently examined in an explicit manner in the training of new researchers, in handbooks and in investigative practice. In general, emphasis is placed on the importance of the laws and rules of formal logic that consider contradictions as a negative needing to be eliminated in order to conserve the scientific validity. In reality, the two logics are not mutually exclusive; they are complementary in contemporary scientific investigations. The objective of this article is to offer different arguments and recommendations contributing to the application of dialectic logic in a rigorous, pertinent and systematic manner and could serve as a useful reference material for the design and development of educational research.

Key words: DIALECTICAL LOGIC, CONTRADICTION, EDUCATIONAL RESEARCH

\footnotetext{
${ }^{1}$ Profesor universitario. Doctor en Ciencias Psicológicas. Director del Centro de Estudios sobre Ciencias de la Educación Superior (CECES) de la Universidad de Holguín Oscar Lucero Moya, Cuba.
}

Dirección electrónica: eortiz@ict.uho.edu.cu

Artículo recibido: 28 de octubre, 2010

Aprobado: 28 de julio, 2011 


\section{Introducción}

En toda investigación educativa se revelan, de manera directa o latente, varias contradicciones dialécticas que reflejan la esencia del objeto de estudio en su unidad y negación a la vez, como manifestación fidedigna del constante cambio y transformación en que se encuentran los procesos y fenómenos objetivos y subjetivos que ocurren en el campo educativo.

Sin embargo, en la formación de profesionales en investigación educativa, en los manuales que se editan sobre el tema y en la propia práctica investigativa, no es frecuente encontrar el abordaje explícito de la lógica dialéctica y su correspondiente aplicación. Por el contrario, es común encontrar recomendaciones y exigencias basadas solamente en las leyes y reglas de la lógica formal, las cuales son imprescindibles pero insuficientes porque en la realidad investigativa ambas lógicas son necesarias. En verdad, los principios y leyes de la lógica dialéctica no están reñidos con la lógica formal, ni son excluyentes, por el contrario, su complementariedad deviene en una exigencia contemporánea para las investigaciones educativas.

Como afirma Gil la lógica formal tiene una validez innegable porque surgió junto con la dialéctica espontánea y convivió con ella por su utilidad hasta el presente en la búsqueda de los conocimientos científicos (Gil, 2007).

La primera ley de la lógica formal es de la identidad, que es necesaria cuando se definen los objetos, procesos y fenómenos de la realidad, y en los elementos esenciales radican sus diferencias con los demás. Esta ley nunca perderá pertinencia porque las cosas tienen una identidad relativa y el conocimiento de sus límites constituye un fundamento importante en el proceso cognitivo. Pero su utilidad se mantendrá siempre que se analice al margen del tiempo, de su evolución interna y de sus relaciones con los demás fenómenos.

La segunda ley es de la no contradicción, la cual precisa que la identidad de algo niega su no identidad, la cual aporta la necesaria la certidumbre en la determinación de las diferencias esenciales entre los objetos de la realidad, aunque también al margen del tiempo y de los cambios internos de dicho objeto.

La ley del tercero excluido establece que no pueden tener razón dos afirmaciones que digan lo contrario, pues una de las dos debe ser falsa, ya que es imposible que coexistan de 
manera sincrónica. Y la ley de la razón suficiente afirma de manera irrebatible que toda razón lógica ha de estar demostrada en un número de acciones prácticas que la confirmen.

No se puede investigar con rigurosidad científica si no se aplican de manera consciente y sistemática las leyes de la lógica formal, pero su aplicación unilateral refuerza su carácter limitado al considerar a la contradicción como algo negativo, que se debe evitar o eliminar en aras de conservar el rigor investigativo.

En la investigación educativa la aplicación del método analítico proporciona un conocimiento científico veraz, pero a la larga restringido porque necesita del método dialéctico. En realidad, el primero está subordinado al segundo por ser este más general.

Cuando se pretende penetrar en la complejidad del objeto de investigación educativa, en sus contradicciones externas e internas, en los cambios evolutivos a través del tiempo y en sus estrechas interacciones y relaciones con otros fenómenos, se evidencia la necesidad de apelar también a los aportes de la lógica dialéctica que permiten reflejar con más nitidez esta pluralidad.

El objetivo de este ensayo es ofrecer varios argumentos y recomendaciones que contribuyen a la aplicación rigurosa, necesaria y sistemática de la lógica dialéctica en las investigaciones educativas. Los años de experiencia del autor como investigador en la Universidad de Holguín (Cuba) le han permitido sistematizar, teóricamente, los contenidos abordados, a partir de la revisión del estado del arte del tema, del intercambio con otros colegas, de su participación en tribunales permanentes de defensas de tesis doctorales en ciencias pedagógicas y de la realización de cursos de posgrado para la profesionalización de jóvenes investigadores e investigadoras en investigación educativa.

\section{Desarrollo}

\section{El enfoque dialéctico}

Como afirma Castro, la dialéctica es la ciencia de las leyes más generales del desarrollo de la naturaleza, la sociedad y el pensamiento. Brinda una concepción abarcadora de la realidad objetiva y subjetiva en su conjunto, en su movimiento y desarrollo, esclarece sus orígenes y sus fuerzas motrices. De manera que la dialéctica es, a la vez, una concepción del mundo, una epistemología, un método de conocimiento y de transformación de la realidad concreta (Castro, 2000). De acuerdo con Gil, la dialéctica permite conocer e interpretar con gran 
valor práctico, como arma transformadora de la realidad social y se expresa también en el arte a través de la creatividad (Gil, 2007).

Como afirma Gil, desde el punto de vista histórico las primeras manifestaciones culturales humanas desarrollaron un pensamiento holista, totalizador, caracterizado por un fuerte contenido dialéctico y sistémico, que interpretaba la realidad como un todo, en el que las partes interactúan en equilibrios inestables que varían por sus tensiones internas y por los cambios globales, de modo que un defecto o un exceso en una parte termina repercutiendo en la totalidad y viceversa. Esta visión holista que ha venido evolucionando hasta el presente se sustenta en la unidad de los contrarios por sus fuerzas centrífugas unidas en lucha con las fuerzas centrípetas holistas (Gil, 2007).

Kohan destaca que el núcleo central de la lógica dialéctica, desde Hegel hasta Lenin, contiene: abarcar y estudiar la totalidad de los aspectos, junto con las vinculaciones e intermediaciones, estudiar los fenómenos en su historicidad, en su automovimiento y desarrollo, el carácter insustituible de la práctica humana y el carácter concreto de la verdad (Kohan, 2003).

Kopnin, Andreiev y Gil, de este núcleo central, derivan los siguientes principios que le sirven como fundamentos generales (Kopnin, s/f; Andreiev, 1984 y Gil, 2007):

- Principio de la concatenación universal de los fenómenos

- Principio del historicismo

- Principio del desarrollo

- Principio de la unidad de lo histórico y lo lógico

- Principio de la ascensión de lo abstracto a lo concreto

- Principio de la bifurcación de la unidad y conocimiento de las partes contradictorias del objeto

- Principio de la unidad del análisis y la síntesis

- Principio del desarrollo desigual y combinado

llénkov precisa que en la lógica dialéctica la contradicción, como unidad concreta de opuestos que se excluyen mutuamente, constituye su categoría central (lliénkov, 1977). Por tanto, la unidad y lucha de contrarios es su ley más importante porque establece la fuente del desarrollo en la naturaleza, la sociedad y en el pensamiento. Ella expresa que todos los 
fenómenos y procesos objetivos y subjetivos poseen aspectos y tendencias contradictorias que están en pugna entre sí.

Por su parte, Kopnin destaca que toda contradicción posee un doble aspecto: externo e interno. Las contracciones internas son consideradas como esenciales porque se erigen en la fuente del desarrollo, desde el punto de vista histórico primero aparecen las externas y posteriormente las internas. Las contradicciones internas de las formas del pensamiento constituyen su condición fundamental de existencia (Kopnin, s.f.).

Las contradicciones dialécticas son dinámicas por su esencia y la agudización de los elementos opuestos dentro del objeto provoca su superación a un nuevo nivel cualitativo y no su solución, si se es consecuente con la lógica dialéctica, ya que si se resolvieran se detendría el desarrollo.

Como resultado del proceso constante del desarrollo, en un momento determinado de la evolución se produce un incremento interno de los contrastes por la consunción de lo viejo y el nacimiento de lo nuevo, que agudiza las contradicciones dentro del objeto, de acuerdo con Kopnin y Rosental (Kopnin, s.f.; Rosental, 1962).

El enfoque dialéctico concibe al desarrollo como un proceso complicado, sutil y profundo, que implica transformaciones cualitativas, el paso de las acumulaciones cuantitativas a una nueva cualidad, donde las anteriores aparecen en una forma básicamente nueva. Koroliov valora que esta concepción es la que brinda la clave del automovimiento de todo lo existente, de los saltos, del receso de la paulatinidad, de la transformación en el contrario, de la anulación de lo viejo y el surgimiento de lo nuevo (Koroliov, 1977).

El principio del desarrollo es uno de los fundamentales, el cual expresa que los fenómenos del mundo no solo tienen movimiento, sino que evolucionan de estadios inferiores a estadios superiores, según Kopnin (Kopnin, s.f.).

Pero estas consideraciones teóricas generales del pensamiento filosófico deben ser coherentemente derivadas al nivel teórico particular de las ciencias y en específico a las ciencias de la educación, con la obligada argumentación lógica mediante un razonamiento predominantemente deductivo. 


\section{La comprensión dialéctica del proceso educativo}

En las concepciones educativas contemporáneas se encuentran dos grandes escuelas que hacen de la contradicción dialéctica un pilar importante en sus elaboraciones teóricas:

\subsection{La escuela de epistemología genética de Piaget (1896-1980)}

En Latinoamérica está difundida y aplicada su pedagogía operatoria en la educación, lo que no ha ocurrido así en Cuba por determinados condicionantes sociales e históricos que no es posible abordar en el presente ensayo. Es innegable que la concepción piagetiana constituye un referente obligado cuando se abordan problemas educativos por sus valores intrínsecos y por la influencia que ha ejercido en teorías posteriores.

La teoría piagetiana es compleja, plena de ideas y matices bien originales y en constante perfeccionamiento. Los experimentos de Piaget con niños (estudios de caso) y las elaboraciones teóricas que extrajo de ellos dan fe de su privilegiada inteligencia, de su sentido común y su originalidad. Cuando se estudia cualquiera de sus obras se destaca su profunda preparación multidisciplinaria y su ferviente vocación humanista. En su complicada teoría son notorias las influencias de su formación como biólogo, epistemólogo y psicólogo, aunque también de la lógica, matemática, cibernética, informática y sociología.

Como uno de los grandes genetistas dentro de la historia de la psicología, en sus concepciones se refleja un enfoque lógico dialéctico, punto de vista compartido con Valera (1995), con una fuerte influencia de la biología, pues considera que la psicología es una continuación de esta ciencia natural por buscar ambas la explicación de la vida.

La dialéctica se refleja en el constante equilibrio-desequilibrio y asimilación-acomodación de las estructuras cognitivas como una regularidad evolutiva humana, fenómenos excluyentes, y a la vez unitarios, que al entrar en contradicción promueven el desarrollo. Sus más destacados seguidores han enriquecido sus aportes pero sin afectar el núcleo duro referido al desarrollo psicológico como eterno proceso contradictorio, lo cual aparece de manera explícita en varias de sus obras (Piaget, 1970, 1974, 1975, 1981). Por tanto, todo investigador que asuma como soporte teórico esta escuela debe partir de las contradicciones dialécticas como sustento de sus indagaciones. 


\subsection{La escuela histórico cultural de Vigotsky (1896-1934)}

Por determinadas condiciones sociales e históricas también, la concepción vigotskiana y sus obras claves son familiares a la educación cubana y no bien conocidas ni aplicadas por el resto de los países latinoamericanos, debido a determinadas causas y condiciones históricas que tampoco pueden ser abordadas en este trabajo. En la actualidad goza de gran popularidad en los medios académicos de Europa, especialmente en España y también en los Estados Unidos.

En las consideraciones de Vigotsky queda bien delimitado su enfoque dialéctico materialista en la unidad de lo interno y lo externo, el proceso de interiorización, como tránsito de lo interpsicológico a lo intrapsicológico, la unidad y diferencia entre lo cognitivo y lo afectivo, del pensamiento con el lenguaje, así como el concepto de zona de desarrollo próximo (Vigotsky, 1980, 1987, 1991).

Sus más fervientes seguidores han sido consecuentes con esta concepción y la han enriquecido desde el punto de vista teórico y metodológico en su aplicación sistemática a la educación, específicamente en el proceso de enseñanza-aprendizaje y en la concreción de la utilidad teórico-práctica del concepto de zona de desarrollo próximo en diferentes niveles de enseñanza.

Ambas escuelas desarrollaron sus respectivas posiciones teóricas desde la psicología con sus específicas categorías, principios, regularidades y metodologías como núcleos fundamentales, sobre la base de la investigación científica desarrollada por sus líderes y continuadores, de acuerdo con sus peculiares posturas filosóficas, con una amplia, abundante y exitosa aplicación en el campo educativo.

Por tanto, el sustento teórico particular del enfoque dialéctico en las investigaciones educativas está sólidamente fundamentado en estas dos concepciones, incluyendo la continuidad que ha tenido a través de sus seguidores y constituye un antecedente importante, pero se hace necesario abundar más en este sentido.

\subsection{La dialéctica del proceso de enseñanza-aprendizaje}

La dialéctica en la educación se evidencia en el proceso de enseñanza- aprendizaje porque ambos fenómenos son totalmente contrarios, la esencia de uno es lo contrapuesto del otro, pero se presuponen: enseñar es lo contrario de aprender y viceversa, ya que cuando se 
enseña se está produciendo un proceso de exteriorización y cuando se aprende ocurre un proceso de interiorización, pero en una unidad condicionada: se exterioriza para que exista interiorización y esta sucede cuando hay una exteriorización previa.

La agudización de las contradicciones entre el enseñar y aprender provoca que de la coexistencia de ambos se pase la trasmutación de uno en el otro, tal como afirma Machado, el que enseña termina aprendiendo y el que aprende culmina enseñando, y de esta manera se promueve el desarrollo cualitativo de dicho proceso (Machado, 2006).

Del carácter dialéctico entre el enseñar y el aprender se derivan los demás rasgos comúnmente aceptados por la comunidad científica, tales como el carácter integrador, bilateral, problematizador, contextual e interdisciplinario, y como consecuencia desarrollador, pero no siempre asumidos de manera consecuente desde lo teórico general hasta lo particular.

Los profesores universitarios constatan primero, de manera intuitiva y después académica, que en su labor docente las contradicciones son omnipresentes, a veces de forma explícita y en ocasiones de manera latente. Las mejores experiencias profesionales de los docentes se encuentran en la enseñanza de contenidos que fueron problematizados y que les provocaron conflictos internos a sus estudiantes, y para superarlos tuvieron que esforzarse intelectualmente (muy motivados), lo cual forma parte también de sus vivencias estudiantiles en el pregrado.

Para que la enseñanza promueva un aprendizaje real y efectivo en los estudiantes tiene que suscitar conflictos cuya agudización promueva un cambio interno en ellos y hasta en los propios profesores, lo cual ocurre de manera regular a veces sin la plena conciencia de ello por parte de los actores fundamentales de este proceso.

La aceptación implícita de esta realidad antagónica sin los ineludibles argumentos científicos, lacera cualquier aspiración de perfeccionar el proceso de enseñanza-aprendizaje universitario en la era de la sociedad de la información y del conocimiento, ya que se dejaría al voluntarismo y a la improvisación la utilización de contradicciones docentes, con el peligro de obtener resultados totalmente contrarios a los pretendidos, como ha ocurrido siempre que se ha intentado aplicar innovaciones didácticas sin la preparación profesional previa.

El proceso de enseñanza-aprendizaje debe promover cambios internos en sus protagonistas, los cuales se producen en sus respectivas personalidades, pero no cualquier modificación o corrección sino aquellos que estimulen el desarrollo, es decir, riqueza, crecimiento, progreso y bienestar individual, por tanto, debe ser un proceso desarrollador. 
El término desarrollador adquiere una connotación importante porque es algo más que un cambio, es un proceso evolutivo a un estadio cualitativamente superior de una manera contradictoria, discontinua, irregular y diferenciada. El desarrollo es concebido como un resultado de la agudización y resolución de las contradicciones que ocurren dentro del proceso de enseñanza-aprendizaje, y que se deben reflejar en la conciencia de sus protagonistas: profesores y estudiantes.

Como plantea Kostiuk, solo interiorizando, provocando en el individuo tendencias contradictorias que luchen entre sí se convertirán en la fuente de su actividad, dirigida a resolver la contradicción interna mediante la elaboración de nuevos procedimientos de conducta. Este autor afirma de manera certera que el carácter dialéctico del desarrollo encuentra su expresión en el proceso formativo de la personalidad y una de las contradicciones internas fundamentales se manifiesta entre las nuevas necesidades que surgen y el nivel alcanzado en las vías y dominios para satisfacerlas, por lo que estimulan a aprender nuevas formas de conducta y nuevos procedimientos para la acción o modos de actuar (Kostiuk, 1986). Tolstij precisa que el desarrollo de la personalidad es, en principio, infinito, ya que es el modo fundamental de existencia de las personas (Tolstij, 1989).

Morin sentencia que:

Toda evolución es el fruto de la desviación cuyo desarrollo transforma el sistema donde ella misma ha nacido: ella desorganiza el sistema reorganizándolo. Las grandes transformaciones son morfogénesis, creadoras de formas nuevas que pueden constituir verdaderas metamorfosis. De todas formas, no hay evolución que no sea desorganizadora-reorganizadora en su proceso de transformación o de metamorfosis. (2000, p. 63)

Por tanto, se puede afirmar que este carácter contradictorio constituye la vía normal, efectiva y cotidiana en que se desarrolla el proceso de enseñanza-aprendizaje, pero aceptarlo o intuirlo no basta, se hace necesario fundamentarlo desde determinadas posiciones teóricas para que la rigurosidad científica sea lo que predomine en su comprensión.

Como la contradicción dialéctica expresa la fuente interna del desarrollo de los fenómenos, el proceso de enseñanza-aprendizaje promueve la agudización de varias contradicciones desarrolladoras, las cuales tienen carácter subjetivo y objetivo a la vez por su existencia ontológica, pueden ser de diversa naturaleza: externas e internas, en dependencia de los elementos que participan en ellas. 
Las contradicciones externas reflejan la situación social del desarrollo debido a la confrontación que aparece entre las exigencias sociales y las posibilidades individuales de profesores y estudiantes de satisfacerlas, y ocurren a un nivel interpsicológico al manifestarse a través de la comunicación interpersonal y las relaciones sociales.

Las contradicciones externas en el proceso de enseñanza-aprendizaje se pueden manifestar entre:

- Las exigencias del profesor y el nivel de desarrollo cognitivo y afectivo de los estudiantes.

- Las exigencias de los diseños curriculares de las carreras, expresadas en sus objetivos, y el nivel de desarrollo cognitivo y afectivo de los estudiantes.

- Las exigencias de los diseños curriculares de las carreras, manifestadas en sus objetivos, y el nivel de desarrollo de las competencias profesionales de los docentes.

- Las exigencias interdisciplinares del desempeño profesional, contenidas en los diseños curriculares y la formación disciplinar previa de los docentes.

La propia esencia compleja del acto didáctico condiciona que estas contradicciones coexistan como posibilidad porque se pueden manifestar total o parcialmente, en dependencia del contexto educativo, cultural y social, así como del nivel de desarrollo personal de estudiantes y profesores.

Las contradicciones en su manifestación interna, por su importancia y nivel de esencia, resultan más difíciles de fundamentar, ya que estas ocurren a un nivel intrapsicológico, es decir, dentro de la subjetividad, provocando un conflicto psicológico, con carácter consciente o no, que los afecta en su integridad como sujetos y como personalidades. Las contracciones internas son esenciales porque se erigen en la fuente del desarrollo de la personalidad de ambos, pero desde el punto de vista histórico primero aparecen las externas que promueven la aparición de las internas.

Estas contradicciones se pueden manifestar entre:

- El desarrollo cognoscitivo de los profesores y el nivel motivacional hacia su profesión docente.

- El desarrollo cognoscitivo de los estudiantes y el nivel motivacional hacia sus carreras.

- La dependencia y la independencia cognoscitiva del estudiante (L. Reyes y otros, 2002)

- Las necesidades, aspiraciones y motivos de los estudiantes, expresadas como conflictos de motivos extrínsecos e intrínsecos asociados a su futura profesión. 
- Las necesidades, aspiraciones y motivos de los profesores, reflejados como conflictos de motivos extrínsecos e intrínsecos asociados a su profesión docente.

- La reflexión individual de los estudiantes para la aceptación de sus insuficientes conocimientos, habilidades, estrategias y estilos de aprendizaje para enfrentar exitosamente el proceso de enseñanza-aprendizaje universitario.

- La reflexión individual de los profesores para aceptar su insuficiente preparación pedagógica ante las crecientes exigencias del proceso de enseñanza-aprendizaje universitario.

Es decir, que las contradicciones pueden ocurrir entre lo cognitivo y lo afectivo de la personalidad, dentro de los propios fenómenos cognitivos y dentro de la fenomenología afectiva, lo cual refleja la complejidad de ellas y la dificultad para determinarlas. Todas ellas pueden estimular el desarrollo si son bien conducidas y encauzadas profesionalmente por parte de los profesores y los estudiantes.

Las contradicciones internas dentro del componente afectivo ocurren entre las necesidades, aspiraciones y los motivos de profesores y estudiantes expresados como conflictos de motivos, en el que participan motivos extrínsecos e intrínsecos asociados a su profesión. Estas contradicciones motivacionales pueden erigirse en fuentes del desarrollo si son bien guiadas profesionalmente.

Las contradicciones internas dentro del componente cognitivo ocurren a través de la reflexión individual de los estudiantes en la aceptación de sus insuficientes conocimientos, habilidades, estrategias y estilos de aprendizaje para enfrentar exitosamente el proceso de enseñanza-aprendizaje universitario. En el caso de los profesores sería la toma de conciencia de sus limitados conocimientos y habilidades profesionales, estrategias didácticas y estilos de enseñanza para dirigir con profesionalidad el proceso de enseñanza-aprendizaje en la educación superior.

La mera existencia de contradicciones externas e internas no promueven per se, ni automáticamente el desarrollo, tiene que producirse una agudización de ellas a partir de la ocurrencia de determinadas causas y condiciones sociales, pedagógicas y psicológicas, en las cuales la toma de conciencia de los sujetos implicados y su decisión personal de asumirlas juegan un papel determinante. Majmutov considera que este es un proceso complejo porque el 
reflejo de las contradicciones dialécticas en la conciencia de las personas solo puede ocurrir al nivel del pensamiento teórico que se realiza sobre la base del lenguaje (Majmutov, 1983).

Las contradicciones internas son las que propician el desarrollo y no las externas, aunque estas siempre anteceden, son imprescindibles que ocurran y propician la aparición de aquellas. El carácter contradictorio del proceso de enseñanza-aprendizaje debe determinar la aparición de contradicciones externas e internas en la personalidad, pero ello no ocurre siempre de manera inexorable.

González (1995a) valora que si bien las contradicciones están presentes de forma permanente en la vida de las personas, no toda contradicción deviene fuerza motriz del desarrollo, sino solo aquellas que producen una tensión emocional particular, es decir, que poseen el suficiente potencial movilizador para ser considerada como tal y comprometer al sujeto con una respuesta que puede colocarlo ante una situación social cualitativa diferente, donde entren en juego nuevos recursos que den lugar a un cambio estable en su configuración subjetiva.

Este mismo autor refiere que la naturaleza subjetiva de las contradicciones puede ser diversa, algunas de la cuales coexisten en el espacio subjetivo del sujeto y lo afectan simultáneamente, algunas de ellas se concientizan, implicando de forma directa su intencionalidad; otras se expresan en vivencias cuya naturaleza muchas veces no comprende, por lo que el tipo de vivencia que producen las contradicciones constituyen elementos de desarrollo o de daño para la persona. Las vivencias negativas resultantes de la contradicción no concientizada es enmascarada de una forma defensiva, lo cual puede provocar serios quebrantos a la personalidad y a la salud del individuo.

Muchas de las contradicciones que el sujeto enfrenta a lo largo del desarrollo devienen períodos críticos definidos como crisis, que constituyen una regularidad del proceso no identificable con etapas específicas, sino con momentos cualitativos particulares del sujeto en desarrollo (González, 1995a).

De manera precisa y aguda González (1995b) se refiere a que las contradicciones son siempre una fuente potencial del desarrollo. Sin embargo, que adquieran o no este carácter en una forma concreta de este proceso va a depender mucho de cómo se integra en el complejo conjunto de fuerzas que caracterizan el proceso de desarrollo en el momento en que la contradicción aparece. Composición de fuerzas dentro de las cuales hay que considerar tanto los factores subjetivos como los objetivos. Cualquier contradicción puede conducir a una crisis, 
cuya resultante sea un nuevo momento en este proceso, o bien un momento de involución del mismo que puede llevarlo a su destrucción.

Continúa este mismo autor argumentando que las contradicciones, tanto a nivel social como individual, son momentos complejos de confluencia de fuerzas. Una de ellas es el sentido subjetivo que la situación tiene para los sujetos que la enfrentan. Este no va a depender linealmente de los factores objetivos que afectan a los implicados, sino de su propia historia, a través de la cual, mediante los diferentes sistemas de relaciones relevantes en que se han desarrollado, aparecen formas diferenciadas de subjetividad, social e individual, que resultan decisivas en la formación del sentido subjetivo de cualquier situación social.

La distinción teórica entre sujeto y personalidad permite explicar mejor por qué la contradicción en sí misma no promueve el desarrollo personal, es decir, que no es suficiente la conciencia de encontrarse ante una situación personal conflictiva, debe además, asumirse el reto que deviene de ella y enfrentarla con la intención de trascenderla. La contradicción entre el sujeto y la personalidad aparece primero de manera inconsciente y no siempre llega a concientizarse por su propia esencia compleja. El carácter no consciente de este fenómeno al inicio se refiere a que la persona vivencia una situación contradictoria que le está afectando, pero no ha podido determinar las verdaderas causas.

F. González (1995a) delimita que personalidad y sujeto, sin ser una misma cosa, constituyen íntegramente el sistema conocido como subjetividad individual. A ambos hay que concebirlos en una representación dialéctica para poderlos aprehender, el sujeto se encuentra permanentemente en una situación interactiva dentro de sistemas diversos y simultáneos de relaciones sociales, las que exigen múltiples decisiones personales con consecuencias y sentidos diferentes para él.

Este autor lo define como el individuo concreto, portador de personalidad que posee como características esenciales la de ser actual, interactivo, consciente y volitivo, el cual no puede sustraerse a su integridad individual actual como condición de su expresión personalizada. El individuo como sujeto psicológico intenta organizar de forma consciente la simultaneidad de vivencias que experimenta y construye, a través de la creación de un sistema de representaciones conscientes sobre el medio, sus relaciones interpersonales y sobre sí mismo.

El sujeto es un mediatizador activo de las influencias sociales y de su repercusión en la personalidad, lo cual permite explicar comportamientos simuladores o aparentes para no entrar 
en contradicción con las exigencias del proceso pedagógico, así como el carácter no lineal ni automático del valor intrínseco de las actividades educativas que se realizan.

Por tanto, se puede afirmar que el ser humano es la síntesis de la relación sujetopersonalidad. La personalidad aporta el sistema de recursos psicológicos que se han ido configurando a través de sus vivencias en las relaciones con los demás y consigo mismo, a partir de una estrecha relación entre elementos estructurales y funcionales. Y el sujeto es un mediador, enfrenta conflictos y contradicciones, se plantea objetivos y regula volitivamente la acción. Tiene un carácter más funcional y situacional que la personalidad y no se organiza en una estructura.

Se puede llegar racional o afectivamente a descubrir de manera individual o con la ayuda de otros las contradicciones externas o internas en que se encuentran inmersos estudiantes y profesores, lo cual es un logro importante y necesario, pero insuficiente porque tienen además, que aceptarlas volitivamente (como sujeto) como reto individual y actuar en consecuencia para que estimulen el desarrollo personal.

Por ejemplo, un alumno que decide (como sujeto) estudiar una carrera universitaria para ser un futuro profesional de nivel superior con todas las potencialidades intelectuales para serlo; pero sus estilos de aprendizaje (personalidad) son ineficaces para enfrentar y resolver las exigencias de las disciplinas y asignaturas que forman parte del plan de estudios. Para que se convierta en una contradicción que genere desarrollo este alumno debe, ante todo, concientizar cuáles son sus insuficiencias y lograr, con sus esfuerzos personales y la ayuda de compañeros y profesores, el perfeccionamiento de dichos estilos para estar a la altura de un alumno universitario en la actualidad. Si intenta continuar utilizando sus estilos ineficaces de aprendizaje se abocará ante una contradicción no dialéctica que lo llevará a su reprobación o a la deserción, lo que no permitirá cumplir con sus aspiraciones profesionales.

Les corresponde a los profesores, como dirigentes del proceso de enseñanzaaprendizaje, descubrirles a sus estudiantes estas contradicciones para entonces motivarlos a asumirlas, con los niveles de ayuda necesarios e individualizados $y$, de acuerdo con su desarrollo real, promover su desarrollo potencial.

Otro ejemplo, puede ser el de un profesor universitario que imparte docencia en la carrera en que se graduó, pero carece de la profesionalización pedagógica necesaria que le permita enfrentar con éxito las crecientes demandas de los nuevos diseños curriculares y las exigencias a los docentes en este nivel de enseñanza. Él vivencia esta contradicción y como sujeto decide 
afrontar el reto y buscar por diferentes vías esta preparación: estudio individual de bibliografía actualizada sobre el tema, superación posgraduada y asesoramiento con profesores de experiencia, por lo que está contribuyendo al desarrollo de su personalidad. Si por el contrario, opta por superarse solo en los contenidos de su especialidad, adopta una actitud hipercrítica hacia las demandas actuales y evade sus deficiencias formativas, es evidente que como sujeto no decide resolver su problema y enfrentará otras contradicciones no dialécticas que no coadyuvarán a su desarrollo profesional.

El proceso de profesionalización docente, en sus múltiples modalidades y variantes, debe contribuir al enfrentamiento de dichas contradicciones y al incremento de la profesionalidad de los claustros universitarios, para que asuman responsablemente (como sujetos) y con satisfacción el desarrollo de sus competencias profesionales en las condiciones actuales del desarrollo de la educación superior.

\section{Las contradicciones dialécticas en las investigaciones educativas}

Desde una óptica investigativa, lliénkov afirma que la contradicción en las determinaciones teóricas del objeto es, ante todo, un hecho que se reproduce de modo constante con el movimiento de la ciencia (lliénkov, 1977). En el proceso investigativo, al tratar de delimitar la situación problémica y el problema científico, se identifica primeramente una contradicción en su manifestación externa que contiene al objeto, relacionado con el efecto y la forma, aunque no necesariamente toda contradicción de esta naturaleza engendra un problema científico, de acuerdo con lo planteado por Machado (2006).

La manifestación externa de la contradicción es más evidente, observable y asequible que su manifestación interna, por eso es que aquella aparece contenida en la situación problémica, pero para lograr la verdadera aprehensión de esta, como parte de la delimitación del problema científico, se necesita de un proceso posterior de carácter subjetivo de abstracción, complejo y mediato para llegar a las causas y al contenido, siguiendo el razonamiento de Machado (2006). La esencia del concepto problema, como categoría de la lógica dialéctica, consiste en que en la investigación científica se revela la existencia de una contradicción dialéctica en el objeto, la cual debe precisar el investigador. Majmutov delimita que el problema, como categoría psicológica, refleja a su vez, las contradicciones dentro del proceso de conocimiento del objeto 
por el sujeto y su descubrimiento interno les corresponde a los profesores en su labor docente con los estudiantes (Majmutov, 1983).

Machado resalta que la contradicción científica debe ser considerada como tal cuando posee relevancia para la comunidad científica respectiva, posee actualidad social y en los conocimientos acopiados previamente no se encuentran respuestas "satisfactorias"; ya que al valorar el estado del arte, como parte de la fundamentación teórica del problema, se pueden descubrir soluciones pero que no son viables para eliminarlas en el contexto donde se generan (Machado, 2006).

Fuentes concibe a las contradicciones dialécticas en la dinámica del proceso investigativo, resaltando su valor en la revelación del problema y del objeto de investigación, así como en todo el proceso indagatorio como reflejo del antagonismo existente entre lo objetivo-subjetivo, lo empírico-teórico y entre la preservación de la cultura y su desarrollo (Fuentes, 2010).

El principio dialéctico del desarrollo desigual y combinado sostiene que en todo proceso los ritmos de sus partes son desiguales, pero en su desigualdad existe una medida combinada que, sin embargo, no garantiza que los diversos ritmos de las partes diferentes del proceso incidan en el resultado último, acelerándolo o retrasándolo. Gil afirma que la irregularidad, es decir, la dialéctica surge cuando una medida es tan desigual y tan persistente en su diferencia que puede provocar cambios en el proceso en su totalidad, abriendo otro nuevo (Gil, 2007).

En la investigación educativa es eficaz este principio cuando se determinan las contradicciones externas e internas del objeto, ya que son una manifestación latente o manifiesta de sus partes que tienen un ritmo de desarrollo diferente y en su evolución agudizan las diferencias, y por tanto, las oposiciones. Resulta una tarea ardua, complicada, y a la vez efectiva, la determinación de aquellos elementos contradictorios que resultan significativos y con disparidades en su desarrollo, las cuales agudizan las discrepancias y no solo ocurren entre pares sino también entre tríos.

A partir de la determinación de los pares y tríos contradictorios más importantes, de acuerdo con los fines investigativos y con la argumentación correspondiente, deben ser caracterizados en sus rasgos esenciales como condición para la determinación de su evolución.

La realidad educativa está llena de contradicciones dialécticas y relacionadas de manera no lineal ni biunívoca. La misión del investigador es aprehenderlas para estudiarlas esencialmente, pero sería ingenuo asumir que en una sola investigación se logren revelar o 
agotar todas las cualidades esenciales, ni que las relaciones entre la contradicción externa e interna sea la única que exista, en realidad esto no es más que el reflejo del carácter inacabado e infinito del conocimiento.

En la medida que la investigación penetra cada vez más en la esencia de su objeto, se revelan nuevas aristas y dependencias con otros objetos y contradicciones que se convierten en futuros derroteros investigativos, como parte de la necesidad de continuar profundizando en ese sesgo provocado por la contribución teórica y/o práctica resultante.

Como proceso complejo es imposible que se pueda reglamentar o establecer una metodología general y única para determinar contradicciones de esta naturaleza. El investigador nunca lo logrará en su primer intento porque es un proceso paulatino de aproximaciones sucesivas, con aciertos y desaciertos, con avances y retrocesos. Por tanto, no hay ni pueden existir recetas ni soluciones infalibles, ya que siempre dependerá del contenido que se investiga, del objetivo de la investigación y de la tenacidad, experiencia, cultura y profesionalidad de los profesionales en investigación educativa.

Aunque sin las categorías generales de la dialéctica no es posible decir nada acerca de los objetos que la ciencia investiga en la actualidad, pues son categorías lógicas del pensar, se ha pretendido erróneamente utilizarlas en las investigaciones educativas de manera explícita y únicas, tales como forma-contenido, singular-general, necesidad-casualidad, causa-efecto, diacrónico-sincrónico y fenómeno-esencia, lo cual se considera desacertado e improcedente porque se están transgrediendo y confundiendo las diferencias esenciales entre el nivel del conocimiento filosófico y el conocimiento científico particular, aunque desde el punto de vista teórico se pueda establecer una correlación lógica entre estas categorías y las que se puedan plantear al nivel educativo.

Los argumentos para fundamentar las contradicciones dialécticas en el proceso educativo, presupone asumir que en toda teoría científica existen dos niveles de análisis que permiten no solo su comprensión más exacta, sino también una organización más sistemática de sus contenidos con el fin de poderlos utilizar de forma severa y precisa:

- Nivel teórico general: referido a las concepciones filosóficas y epistemológicas que la sustentan y que le sirven de fundamentación.

- Nivel teórico particular: es la concepción científica específica con su objeto, principios, leyes y categorías. 
Por tanto, para la selección de argumentos certeros que permitan fundamentar de manera científica una o varias contradicciones dialécticas dentro del proceso educativo, se puede utilizar una lógica inclusiva que recorra los niveles antes mencionados de la siguiente manera:

- Argumentos teórico generales: referidos a las tres leyes fundamentales de la dialéctica, sobre todo la ley de la unidad y lucha de contrarios y sus categorías, tales como, causaefecto, necesidad-casualidad, histórico-lógico, abstracto-concreto, unidad-diversidad, unitario-sistémico, cuantitativo-cualitativo, simple-complejo, parcialidad-integridad, objetivo-subjetivo e individual-social; que permiten establecer cierta relación genérica con los pares contradictorios que se proponen como fundamentales.

- Argumentos teórico particulares: se encuentran dentro de la concepción específica asumida y, por ejemplo, en el caso de la concepción educativa basada en la concepción vigotskiana, están la situación social del desarrollo, la zona de desarrollo próximo y los conceptos interpsicológico-intrapsicológico, afectivo-cognitivo, instructivo-educativo, actividad-comunicación, interiorización-exteriorización, enseñanza-aprendizaje, motivos extrínsecos-motivos intrínsecos, conocimientos-habilidades-valores, subjetividad individual-subjetividad social, así como sujeto-personalidad.

Pero para lograr con eficacia esta lógica deductiva es recomendable tener en cuenta tres aspectos esenciales:

- La preparación científica del investigador mediante la profundización sistemática y consuetudinaria en las posiciones teóricas generales y particulares que asume, de la cual deberá derivar su estrategia investigativa de manera coherente y consecuente. El conocimiento científico del objeto es un requisito esencial e inexcusable para continuar la investigación, así como los contenidos esenciales de la lógica dialéctica. Nunca será serio que se defienda un autor del cual no se ha realizado un estudio sistemático de sus obras fundamentales, ni criticar a un autor sin haber realizado un estudio similar, como ha ocurrido con las concepciones de Vigotsky y de Piaget.

- La experiencia profesional educativa anterior que le permite al investigador poseer suficientes vivencias profesionales (unidad de lo cognitivo y afectivo) de lo que estudia. Es harto difícil investigar sin una práctica profesional consecutiva y prolongada. Los que se 
aventuran a investigar objetos alejados de su quehacer profesional corren el riesgo de realizar investigaciones que no trascienden el nivel empírico del conocimiento y no aportan resultados verdaderamente científicos y novedosos.

- La contribución práctica de la investigación, derivada del aporte teórico, debe ser llevada a la práctica en cualesquiera de las variantes que se considere, pues las contradicciones emergen de las condiciones concretas de la vida, el investigador las identifica a partir de su preparación teórica y es en dichas condiciones donde en ellas se superan para la promoción del desarrollo y donde se demuestra, en última instancia, la viabilidad y pertinencia de las soluciones científicas propuestas.

\section{Otros principios de la dialéctica en las investigaciones educativas}

Pero la aplicación sistemática de la lógica dialéctica en las investigaciones educativas no solo radica en el descubrimiento de contradicciones, sino también en la aplicación atinada de varios principios de gran nivel de complejidad, pero eficaces cuando son bien empleados, entre los cuales están:

- El principio de la unidad de lo histórico y lo lógico

- El principio de la ascensión de lo abstracto a lo concreto

- El principio de la unidad del análisis y la síntesis

Castro abunda que lo histórico y lo lógico son dos categorías importantes que reflejan el proceso de desarrollo en cuanto a la trayectoria temporal del objeto y su desenvolvimiento lógico. Es decir, lo histórico hace referencia al movimiento del objeto en cuanto a su estructura y función, en su surgimiento y evolución posterior. Y lo lógico, en calidad de elemento derivado o como reflejo de lo histórico, determina los nexos, relaciones, determinaciones y regularidades que caracterizan ese objeto en su estado desarrollado (Castro, 2000)

Entre lo histórico y lo lógico existe una unidad dialéctica porque, como opuestos complementarios, lo histórico contiene a lo lógico debido a que en la evolución del objeto están implícitas sus peculiaridades esenciales y no esenciales, las correlaciones e interdependencias de sus elementos y las características de su desarrollo. Y a la vez, en lo lógico está condensado, de manera latente, la historia del objeto. En resumen, lo histórico es el decursar del objeto en su temporalidad con todo su contenido contingente y necesario, y lo lógico es lo 
histórico despojado de lo casual y sintetizado en lo esencial. A veces se identifica, erróneamente, lo histórico con la cronología de hechos y acontecimientos, lo cual no rebasa el nivel descriptivo.

Kohan valora que el historicismo otorga al método dialéctico carácter crítico y revolucionario porque enfatiza en la negatividad y en lo perecedero de lo actual (Kohan, 2003). Cuando en una investigación se valora el estado del arte del problema científico y su manifestación a través de un tiempo determinado y se distinguen, etapas, periodizaciones, regularidades y tendencias, se está aplicando este principio, con el consiguiente aporte de ideas novedosas que devienen en contribuciones teóricas.

Para que su utilización sea eficaz es importante que las regularidades, tendencias, etapas y períodos propuestos como originales se basen en los que previamente las ciencias de la educación han aportado, así como en los que la historia en general y la historia de la educación en particular han establecido, aun cuando se pretenda criticarlos y perfeccionarlos con el aporte de elementos desconocidos hasta el momento. De lo contrario resultan endebles por una deficiente sustentación debido a la pobre cultura del investigador.

En toda periodización se utilizan indicadores previamente determinados que tienen una determinación lógica, ya que se sustentan en elementos teóricos asumidos previamente por el investigador. Si estos indicadores no pueden ser fundamentados teóricamente, entonces responden a criterios empíricos solamente y se resiente su valor científico.

La determinación de regularidades presupone evidenciar nexos y relaciones que tienen carácter reiterado y necesario, las cuales se precisan a partir de la valoración histórica en la evolución del objeto y acaecen en un descubrimiento lógico que no ha sido aportado previamente por otros profesionales en investigación educativa.

La certeza de haber llegado a nuevas regularidades no es suficiente para afirmar que se han descubierto leyes o principios porque se necesitan muchos más argumentos que la determinación de nexos y relaciones para llegar a ellos. Ante todo habría que demostrar que las leyes y principios existentes no tienen relación alguna con las nuevas regularidades halladas, lo cual sería la base para argumentar la pertinencia de los que se pretenden proponer como alternativos. Todo principio o ley se basa en regularidades, pero no toda regularidad presupone estar en presencia de un principio o una ley.

El descubrimiento de tendencias constituye un resultado científico importante también mediante la aplicación efectiva del principio de la unidad de lo histórico y lo lógico porque refleja 
la dinámica en la evolución del objeto de investigación desde el pasado hasta el presente y su posible proyección futura. La esencia del término tendencia define su contenido dialéctico pues constituye la orientación o dirección hacia algo.

Pero para tener la certeza científica de que se aporta una nueva tendencia, hay que realizar una valoración exhaustiva de las tendencias propuestas por otros profesionales en investigación educativa, en primer lugar para utilizarlas como parte del acervo científico previo que brinda la cultura científica y, en segundo lugar, para delimitar dónde están las diferencias que permitan identificar la existencia de otra diferente. Además, las tendencias en las ciencias sociales no se reflejan linealmente, sino de manera sinuosa, irregular y oscilante, con avances y retrocesos. Las tendencias se descubren desde el pasado, se manifiestan en el presente e influyen en la trayectoria futura del objeto.

Castro define que lo abstracto y lo concreto son categorías dialécticas que están en unidad y contrapuestas por definición, pero se condicionan, ya que una no tendría existencia sin la otra. Lo abstracto es el aspecto o la parte de un conjunto, lo unilateral, lo simple y no desarrollado, lo concreto es lo íntegro, lo multilateral, complejo y desarrollado (Castro, 2000).

Es importante distinguir el uso común del término concreto como sinónimo de específico, particular o determinado del término concreto como categoría, ya que puede provocar confusiones en los profesionales en investigación educativa, al estarse utilizando una misma palabra con dos acepciones dispares. Lo abstracto es lo parcial, lo incompleto, no debe identificarse con lo difícil o inaccesible; y lo concreto es lo total, la síntesis, la determinación de relaciones y determinaciones, la unidad ordenada de lo diverso, no es igual a lo accesible o lo sensible a los sentidos.

Kohan propone las siguientes fases lógicas dialécticas del conocimiento científico en las ciencias sociales, en su tránsito de lo abstracto a lo concreto, de lo simple a lo complejo (Kohan, 2003):

I- Lo real social concreto mediado por la práctica humana que existe fuera de la ciencia, aunque forma parte del objeto de estudio de ella. Esta fase es aprehendida en dos momentos: a partir de la reproducción cognoscitiva de la realidad en la actividad práctica cotidiana y a partir también de la producción o construcción de conocimientos científicos. Ambos momentos pueden coexistir cuando el investigador se encuentra inmerso en la actividad profesional que estudia. 
II - Representación plena que tiene carácter precientífico, constituye todavía una totalidad confusa o caótica porque no se ha logrado una articulación explicativa de forma científica, carece de un orden lógico, predomina el sentido común, provee una certeza sensible de índole intuitiva.

III - Determinaciones abstractas que se logran mediante la definición de los conceptos y categorías, constituye la fundamentación teórica junto con la elaboración de hipótesis y el establecimiento de las jerarquías lógicas.

IV - Totalidad conceptual construida que todavía es abstracta por ser general y no haberse llegado a la particularización, subdivisión y clasificación.

V - Categorías explicativas de carácter novedoso por aportar conceptos científicos no existentes hasta el momento, más densas, específicas y con mayor poder explicativo.

VI - Totalidad concreta explicativa que ocurre en el plano del pensamiento, es lo concreto pensado y posee carácter tendencial por el carácter relativo y no absoluto de la verdad.

Estas fases poseen cierto carácter esquemático porque no ocurren obligatoriamente de manera lineal y no reproducen de forma absoluta el proceso investigativo, ya que siempre dependerá de la lógica particular (inductiva o deductiva) asumida por el investigador. Las fases I y II reflejan la situación problémica (la contradicción externa) que hacen conveniente la investigación; las fases III y IV los presupuestos teóricos que facilitan la formulación del problema científico y su posible explicación-solución y las fases $\mathrm{V}$ y $\mathrm{VI}$ el aporte teórico si constituye un objetivo del proceso indagatorio.

Cuando en la investigación se determinan de manera rigurosa los elementos y cualidades que caracterizan esencialmente al objeto, y a la vez se logran integrar al nivel del pensamiento teórico en su totalidad y complejidad, a través de un concepto, categoría, principio o regularidad, se está aplicando efectivamente este principio. Los aportes científicos, ya sean concepciones, modelos, principios, leyes, regularidades y conceptos, se caracterizan porque logran la concreción del objeto (concreto pensado) como resultado de una abstracción previa.

En la investigación educativa el manejo de elementos abstractos y concretos resulta imprescindible si se aspira al logro de resultados científicos que enriquezcan la teoría científica, y a la vez, que contribuyan de manera efectiva al perfeccionamiento de los procesos educativos, y por tanto, a su optimización y desarrollo. 
El empirismo en las investigaciones educativas ha dejado una huella negativa por su incapacidad de trascender los datos cuantitativos obtenidos, debido a la carencia de una fundamentación teórica previa (lo abstracto) y a la imposibilidad posterior de su interpretación teórica para explicar y proponer solución a los problemas detectados (lo concreto pensado), lo que resiente la efectividad y valor científico de las conclusiones y recomendaciones.

El análisis y la síntesis son categorías dialécticas también porque reflejan al unísono su unidad y diferencias. El análisis es la descomposición a nivel teórico de todos los elementos que integran una totalidad y la síntesis es la recomposición de dicha integridad. Por tanto, el análisis es la antítesis de la síntesis, pero no pueden coexistir de manera independiente uno sin el otro: el análisis parte de una síntesis y ella presupone un análisis previo.

El proceso analítico-sintético constituye uno de los procedimientos básicos del pensamiento, que se encuentra en los fundamentos de los demás procesos intelectuales, desde lo más simples a los más complejos. Como método teórico en la investigación permite la determinación de aquellos elementos y cualidades que integran el objeto sin perder su esencia unitaria e integral y constituyen el soporte de los demás principios y métodos teóricos.

Hay que diferenciar con nitidez el proceso analítico sintético cotidiano que todas las personas utilizan de manera espontánea, como parte de su acervo intelectual, de la unidad del análisis y la síntesis como ley de la lógica dialéctica. En la investigación científica se utiliza como método teórico de manera preconcebida e intencional en aras de la obtención de conocimientos, a partir de la determinación de las partes y el todo del objeto de estudio. La sagacidad del investigador le permite extraerle todo su potencial heurístico en aras de sus objetivos indagatorios.

La abstracción y la generalización, como procesos del pensamiento, constituyen la manifestación suprema del análisis y la síntesis, pero a un nivel superior porque se llegan a revelar contenidos esenciales del objeto. Es decir, la abstracción es la descomposición mental de aquellos elementos del objeto que resultan esenciales en su definición, y a través de la generalización, que resulta la síntesis de esa esencialidad, permite obtener la integración de dicho objeto en su contenido y extensión a través de un concepto o categoría.

En las investigaciones educativas, cuando se aplican métodos teóricos y empíricos, el análisis e interpretación de los datos cualitativos y cuantitativos obtenidos es una manifestación de los procesos analítico-sintéticos y de abstracción-generalización para la determinación de 
aquellos contenidos que son relevantes y/o esenciales, de acuerdo con las intenciones de los profesionales de la investigación educativa.

Del mismo modo, las conclusiones y recomendaciones de la investigación han estado precedidas por todo un proceso analítico y de abstracción de aquellos contenidos considerados como importantes, por lo que es imprescindible sintetizar y generalizar lo esencial, de acuerdo con los objetivos trazados, la hipótesis asumida, el aporte científico obtenido, su novedad y pertinencia. Tan importante es sintetizar-generalizar los resultados científicos en las conclusiones, como resaltar en las recomendaciones cuál debe ser el derrotero que le brinde continuidad a la investigación, para conocer mejor aquellas aristas relacionadas con el objeto, que fueron emergiendo en el proceso indagatorio, y que deviene como un imperativo seguir profundizando en ellas.

\section{Síntesis y reflexiones finales}

La dialéctica como método constituye un recurso de grandes potencialidades heurísticas en las investigaciones educativas porque permite reflejar la complejidad de los fenómenos y procesos formativos en su integridad, en su desarrollo y en sus relaciones e interacciones.

La aplicación rigurosa y efectiva de la lógica dialéctica en las investigaciones educativas resulta imprescindible para el logro de un mayor nivel de esencialidad en el estudio de los objetos de indagación, ya que el desarrollo de la educación contemporánea reclama resultados científicos más abarcadores, que contribuyan a la elevación de la eficiencia de los sistemas formativos en un mundo cada vez más globalizado, donde las tendencias inter y multidisciplinarias van predominando y provocan la obsolescencia de estudios parciales que no tienen en cuenta los acelerados cambios económicos, sociales, políticos y culturales que acontecen en el presente siglo.

Como todo proceso complejo es imposible que se pueda reglamentar o establecer una metodología general y única para su aplicación científica y exitosa. Nunca se logrará de manera eficaz en un primer intento porque definitivamente será un proceso paulatino de aproximaciones sucesivas, con aciertos y desaciertos, con avances y retrocesos. Siempre dependerá del contenido que se investiga, del objetivo de la investigación y, sobre todo, de la cultura general y científica, de la tenacidad, experiencia y profesionalidad de los profesionales de la investigación 
educativa para estar a la altura de la complejidad que entraña el estudio de los procesos educativos en el mundo actual.

Las sociedades modernas, con sus generaciones presentes y futuras, esperan de los investigadores e investigadoras educativas la búsqueda de soluciones científicas y sostenibles para que los sistemas formativos en sus diferentes niveles contribuyan a prepararlos para la vida, sin desconocer sus tradiciones, culturas, creencias e identidades, transitando hacia un mundo mejor, que siempre será posible y deseable.

\section{Referencias}

Castro, Luis. (2000). Diccionario de ciencias de la educación superior. Lima: Seguro editores.

Fuentes, Homero. (2010). La formación de los profesionales en la educación superior. Una alternativa holística, compleja y dialéctica en la construcción del conocimiento científico. Tesis en opción al grado científico de doctor en ciencias. Centro de Estudios de Educación Superior Manuel Gran. Universidad de Oriente. Cuba. (Inédita)

Gil de San Vicente, Iñaki. (2007). La dialéctica como arma, método, concepción y arte. Recuperado el 7 de noviembre de 2010, de www.rebelion.org/noticia.php?id=55787

González Rey, Fernando. (1995a). Comunicación, personalidad y desarrollo. La Habana: Editorial Pueblo y Educación.

González Rey, Fernando. (1995b). Acerca de los social y lo subjetivo en el socialismo, 3, Temas, 93-101.

Iliénkov, Évald V. (1977). Lógica dialéctica. Moscú: Progreso.

Kohan, Néstor. (2003). Marx en su tercer mundo. Hacia un socialismo no colonizado. La Habana: Centro de investigaciones y desarrollo de la cultura cubana Juan Marinello.

Kopnin, Pavel Vasil. (s.f.). Lógica dialéctica. La Habana: Imprenta Universitaria André Voisin.

Koroliov, F. (1977). Lenin y la pedagogía. Moscú: Editorial Progreso.

Kostiuk, G. (1986). El principio del desarrollo en la psicología. En Iliásov, I. y Liaudis, V. (editores) Antología de la Psicología Pedagógica y de las Edades. La Habana: Editorial Pueblo y Educación. 
Machado, Evelio. (2006). Transformación-acción e investigación educativa. Tesis en opción al grado científico de doctor en ciencias. Centro de Estudios de Ciencias de la Educación Enrique José Varona. Universidad de Camagüey, Cuba.

Majmutov, L. I. (1983). La enseñanza problémica. La Habana: Editorial Pueblo y Educación.

Morín, Edgar (2000). Los siete saberes necesarios para la educación del futuro. Colombia: UNESCO-ICFES.

Piaget, Jean. (1970). Naturaleza y método de la epistemología. Buenos Aires: Proteo.

Piaget, Jean. (1974). El criterio moral en el niño. Madrid: Fontanella.

Piaget, Jean. (1975). A dónde va la educación. Barcelona: Teide, S.A.

Piaget, Jean. (1981). Psicología y pedagogía. Barcelona: Ariel.

Rosental, M. (1962). Principios de lógica dialéctica. Montevideo: Pueblos Unidos.

Tolstij, A. (1989). El hombre y la edad. Moscú: Editorial Progreso.

Valera, Orlando. (1995). Estudio crítico de las principales corrientes de la psicología contemporánea. México: Janet Editores.

Vigotsky, Lev. (1987). Historia del desarrollo de las funciones psíquicas superiores. La Habana: Científico-Técnica.

Vigotsky, Lev. (1980). Pensamiento y Lenguaje. La Habana: Pueblo y Educación, Vigotsky, Lev. (1991). Obras Escogidas (Tomo I). España: Visor. 